\title{
GREEN'S RELATIONS ON SEMIGROUPS OF REGRESSIVE TRANSFORMATIONS WITH RESTRICTED RANGE
}

\author{
Kritsada Sangkhanan \\ Department of Mathematics \\ Faculty of Science \\ Chiang Mai University \\ Chiang Mai, THAILAND
}

\begin{abstract}
Let $X^{\prime}$ be a subposet of a poset $X$. Define $P_{R E}\left(X, X^{\prime}\right)$ be the semigroup under composition of all regressive transformations from a subset of $X$ into $X^{\prime}$. Moreover,

$$
T_{R E}\left(X, X^{\prime}\right)=\left\{\alpha \in P_{R E}\left(X, X^{\prime}\right): \operatorname{dom} \alpha=X\right\} .
$$

In 2012, C. Namnak and E. Laysirikul [3] investigated the Green's relations on $T_{R E}(X)=$ $T_{R E}(X, X)$. Now, we aim to extend the result of them by study the Green's relations on the semigroups $T_{R E}\left(X, X^{\prime}\right)$ and $P_{R E}\left(X, X^{\prime}\right)$.
\end{abstract}

AMS Subject Classification: 20M20

Key Words: poset, regressive transformations, restricted range, Green's relations

\section{Introduction}

A partial transformation semigroup is the collection of functions from a subset of $X$ into $X$ with composition denoted by $P(X)$. In addition, the semigroups $T(X)$ and $I(X)$ are defined by

$$
\begin{gathered}
T(X)=\{\alpha \in P(X): \operatorname{dom} \alpha=X\} \\
I(X)=\{\alpha \in P(X): \alpha \text { is injective }\} .
\end{gathered}
$$

We can see that $T(X)$ and $I(X)$ are subsemigroups of $P(X)$. The semigroups

Received: Marhc 26, 2016

Published: June 23, 2016
(C) 2016 Academic Publications, Ltd.

url: www.acadpubl.eu 
$T(X)$ and $I(X)$ are called the full transformation semigroup and the symmetric inverse semigroup, respectively. It is well-known that $P(X)$ and $T(X)$ are regular and $I(X)$ is an inverse semigroup.

Let $X$ be a poset. For $\alpha \in P(X)$, $\alpha$ is said to be regressive if

$$
x \alpha \leq x \text { for all } x \in \operatorname{dom} \alpha .
$$

A transformation semigroup on $X$ is said to be regressive if all of its elements are regressive. Let

$$
\begin{gathered}
P_{R E}(X)=\{\alpha \in P(X): \alpha \text { is regressive }\}, \\
T_{R E}(X)=\{\alpha \in T(X): \alpha \text { is regressive }\} \text { and } \\
I_{R E}(X)=\{\alpha \in I(X): \alpha \text { is regressive }\} .
\end{gathered}
$$

Then $P_{R E}(X), T_{R E}(X)$ and $I_{R E}(X)$ are respectively subsemigroups of $P(X)$.

Some known results of regressive transformation semigroups are as follows. In [7], A. Umar gave a significant isomorphism theorem on full regressive transformation semigroups. For chains $X$ and $Y, T_{R E}(X) \cong T_{R E}(Y)$ if and only if $X$ and $Y$ are order-isomorphic. In 2003, Y. Kemprasit [2] showed that in any regressive transformation semigroup on a poset, its idempotents and regular elements are identical. Moreover, she also characterized when $P_{R E}(X), T_{R E}(X)$ and $I_{R E}(X)$ are regular semigroups and gave the necessary and sufficient condition for $P_{R E}(X), T_{R E}(X)$ and $I_{R E}(X)$ to be eventually regular. In $2012, \mathrm{C}$. Namnak and E. Laysirikul [3] investigated the Green's relations on $T_{R E}(X)$.

Now, we consider subsemigroups of $P_{R E}(X), T_{R E}(X)$ and $I_{R E}(X)$ as follows. Let $X^{\prime}$ be a subposet of $X$, define

$$
\begin{gathered}
P_{R E}\left(X, X^{\prime}\right)=\left\{\alpha \in P_{R E}(X): X \alpha \subseteq X^{\prime}\right\}, \\
T_{R E}\left(X, X^{\prime}\right)=\left\{\alpha \in T_{R E}(X): X \alpha \subseteq X^{\prime}\right\} \text { and } \\
I_{R E}\left(X, X^{\prime}\right)=\left\{\alpha \in I_{R E}(X): X \alpha \subseteq X^{\prime}\right\} .
\end{gathered}
$$

It is clear that if $X^{\prime}=X$, then $P_{R E}\left(X, X^{\prime}\right)=P_{R E}(X), T_{R E}\left(X, X^{\prime}\right)=T_{R E}(X)$ and $I_{R E}\left(X, X^{\prime}\right)=I_{R E}(X)$ which implies that $P_{R E}\left(X, X^{\prime}\right), T_{R E}\left(X, X^{\prime}\right)$ and $I_{R E}\left(X, X^{\prime}\right)$ are the generalization of $P_{R E}(X), T_{R E}(X)$ and $I_{R E}(X)$, respectively.

In 2004, P. Udomkavanich and P. Jitjankarn [1] gave necessary and sufficient conditions for the semigroups $P_{R E}\left(X, X^{\prime}\right), T_{R E}\left(X, X^{\prime}\right)$ and $I_{R E}\left(X, X^{\prime}\right)$ to be regular. In [5], they prove that for chains $X$ and $Y$ and subchains $X^{\prime}$ of $X$ and $Y^{\prime}$ of $Y$, if $T_{R E}\left(X, X^{\prime}\right) \cong T_{R E}\left(Y, Y^{\prime}\right)$, then $X^{\prime}$ and $Y^{\prime}$ are order-isomorphic. In 
[4], they also prove that if $P_{R E}\left(X, X^{\prime}\right) \cong P_{R E}\left(Y, Y^{\prime}\right)\left[I_{R E}\left(X, X^{\prime}\right) \cong I_{R E}\left(Y, Y^{\prime}\right)\right]$, then $X^{\prime}$ and $Y^{\prime}$ are order-isomorphic. Moreover, in [6], they gave necessary and sufficient conditions for $T_{R E}\left(X, X^{\prime}\right)$ and $T_{R E}\left(Y, Y^{\prime}\right)$ to be isomorphic.

In this paper, we aim to extend the result of C. Namnak and E. Laysirikul [3] by study the Green's relations on the semigroups $T_{R E}\left(X, X^{\prime}\right)$ and $P_{R E}\left(X, X^{\prime}\right)$.

Note that in order for $T_{R E}\left(X, X^{\prime}\right)$ to be nonempty, each element of $X$ must lie above some elements in $X^{\prime}$. That is,

$$
\forall x \in X \quad \exists x^{\prime} \in X^{\prime}, \quad x^{\prime} \leq x .
$$

Let us remark here that if $\min X$ exists, then $X^{\prime}$, which satisfies (1), must contain $\min X$. From now on, we assume that the subposet $X^{\prime}$ of $X$ satisfies the property (1) and for each $x \in X$ the element $x^{\prime}$ means the element in $X^{\prime}$ such that $x^{\prime} \leq x$ which exists by the property (1).

For $\alpha \in P(X)$, we denote $\pi(\alpha)$ the composition of $\operatorname{dom} \alpha$ induced by $\alpha$, namely

$$
\pi(\alpha)=\left\{y \alpha^{-1}: y \in X \alpha\right\} .
$$

and define $\alpha_{*}: \pi(\alpha) \rightarrow X \alpha$ by

$$
P \alpha_{*}=x \alpha \text { for each } P \in \pi(\alpha) \text { and } x \in P .
$$

Then $\pi(\alpha)$ is a partition of $\operatorname{dom} \alpha$ and $\alpha_{*}$ is a bijection from $\pi(\alpha)$ onto $X \alpha$.

More generally, we extend the above definition to the partial transformation semigroup with restricted range. Let $X^{\prime}$ be a subset of $X$. Define

$$
P T\left(X, X^{\prime}\right)=\left\{\alpha \in P(X): X \alpha \subseteq X^{\prime}\right\} .
$$

For each $\alpha \in P T\left(X, X^{\prime}\right)$, we define

$$
\tilde{\pi}(\alpha)=\left\{M \cap X^{\prime}: M \in \pi(\alpha) \text { and } M \cap X^{\prime} \neq \emptyset\right\} .
$$

Moreover, we define $\tilde{\alpha}: \tilde{\pi}(\alpha) \rightarrow X \alpha$ by

$$
P \tilde{\alpha}=x \alpha \text { for each } P \in \tilde{\pi}(\alpha) \text { and each } x \in P .
$$

Let $\mathcal{A}$ and $\mathcal{B}$ be collections of subsets of $X$. We say that $\mathcal{B}$ is a refinement of $\mathcal{A}$ or $\mathcal{B}$ refines $\mathcal{A}$ if $\cup \mathcal{A}=\cup \mathcal{B}$ and for every $B \in \mathcal{B}$, there exists some $A \in \mathcal{A}$ such that $B \subseteq A$. 


\section{Green's Relations}

In this section, we characterize Green's relations on $T_{R E}\left(X, X^{\prime}\right)$ and $P_{R E}\left(X, X^{\prime}\right)$.

Theorem 1. Let $\alpha, \beta \in T_{R E}\left(X, X^{\prime}\right)$. Then the following statements are equivalent.

1. $\beta \in \alpha T_{R E}\left(X, X^{\prime}\right)$.

2. For every $P \in \pi(\alpha)$, there exists $Q \in \pi(\beta)$ such that $P \subseteq Q$ and $Q \beta_{*} \leq$ $P \alpha_{*}$.

Proof. Suppose that $\beta \in \alpha T_{R E}\left(X, X^{\prime}\right)$. Then there is $\gamma \in T_{R E}\left(X, X^{\prime}\right)$ such that $\beta=\alpha \gamma$. Let $P \in \pi(\alpha)$. Then $P \alpha_{*}=y$ for some $y \in X^{\prime}$ which implies that $y \gamma \in X \alpha \gamma=X \beta$. Define $Q=y \gamma \beta^{-1} \in \pi(\beta)$. Let $p \in P$. Then $p \beta=p \alpha \gamma=y \gamma$ from which it follows that $p \in y \gamma \beta^{-1}=Q$. So $P \subseteq Q$. We can see that $p \beta=Q \beta_{*}$. Then $Q \beta_{*}=p \beta=p \alpha \gamma \leq p \alpha=P \alpha_{*}$

Conversely, Assume that (2) holds. For each $x \in X \alpha$, there exists unique $P_{x} \in \pi(\alpha)$ such that $P_{x} \alpha_{*}=x$. By (2), there is $Q_{x} \in \pi(\beta)$ such that $P_{x} \subseteq Q_{x}$ and $Q_{x} \beta_{*} \leq P_{x} \alpha_{*}$. Define $\gamma: X \rightarrow X^{\prime}$ by

$$
x \gamma= \begin{cases}Q_{x} \beta_{*} & \text { if } x \in X \alpha ; \\ x^{\prime} & \text { otherwise. }\end{cases}
$$

We can see that $x \gamma=Q_{x} \beta_{*} \leq P_{x} \alpha_{*}=x$ if $x \in X \alpha$ and $x \gamma=x^{\prime} \leq x$, otherwise. Then $\gamma \in T_{R E}\left(X, X^{\prime}\right)$. Let $x \in X$. We obtain $x \alpha \gamma=Q_{x \alpha} \beta_{*}$. Since $x \in P_{x \alpha} \subseteq Q_{x \alpha}$, we get $x \beta=Q_{x \alpha} \beta_{*}=x \alpha \gamma$. Therefore, $\beta=\alpha \gamma$.

Theorem 2. Let $\alpha, \beta \in P_{R E}\left(X, X^{\prime}\right)$. Then the following statements are equivalent.

1. $\beta \in \alpha P_{R E}\left(X, X^{\prime}\right)$.

2. For every $P \in \pi(\alpha), P \cap \operatorname{dom} \beta=\emptyset$ or there exists $Q \in \pi(\beta)$ such that $P \subseteq Q$ and $Q \beta_{*} \leq P \alpha_{*}$.

Proof. Suppose that $\beta \in \alpha P_{R E}\left(X, X^{\prime}\right)$. Then there is $\gamma \in P_{R E}\left(X, X^{\prime}\right)$ such that $\beta=\alpha \gamma$. Let $P \in \pi(\alpha)$. Then $P \alpha_{*}=y$ for some $y \in X^{\prime}$. If $y \notin \operatorname{dom} \gamma$, then $\emptyset=P \cap \operatorname{dom} \alpha \gamma=P \cap \operatorname{dom} \beta$. If $y \in \operatorname{dom} \gamma$, then $y \gamma \in X \alpha \gamma=X \beta$. Define $Q=y \gamma \beta^{-1} \in \pi(\beta)$. Let $p \in P$. Then $p \beta=p \alpha \gamma=y \gamma$ from which it follows that $p \in y \gamma \beta^{-1}=Q$. So $P \subseteq Q$. We can see that $p \beta=Q \beta_{*}$. Then $Q \beta_{*}=p \beta=p \alpha \gamma \leq p \alpha=P \alpha_{*}$ 
Conversely, Assume that (2) holds. For each $x \in X \alpha$, there exists unique $P_{x} \in \pi(\alpha)$ such that $P_{x} \alpha_{*}=x$. Let $A=\left\{x \in X \alpha: P_{x} \cap \operatorname{dom} \beta \neq \emptyset\right\}$. By (2), for each $x \in A$, there is $Q_{x} \in \pi(\beta)$ such that $P_{x} \subseteq Q_{x}$ and $Q_{x} \beta_{*} \leq P_{x} \alpha_{*}$. Define $\gamma: A \rightarrow X^{\prime}$ by $x \gamma=Q_{x} \beta_{*}$. We can see that $x \gamma=Q_{x} \beta_{*} \leq P_{x} \alpha_{*}=x$. Then $\gamma \in P_{R E}\left(X, X^{\prime}\right)$. Let $x \in X$. We obtain $x \alpha \gamma=Q_{x \alpha} \beta_{*}$. Since $x \in P_{x \alpha} \subseteq Q_{x \alpha}$, we get $x \beta=Q_{x \alpha} \beta_{*}=x \alpha \gamma$. Therefore, $\beta=\alpha \gamma$.

Theorem 3. Let $\alpha, \beta \in T_{R E}\left(X, X^{\prime}\right)$. Then $\alpha \mathcal{R} \beta$ if and only if $\alpha=\beta$.

Proof. Assume that $\alpha \mathcal{R} \beta$. Then $\beta \in \alpha T_{R E}\left(X, X^{\prime}\right)$ and $\alpha \in \beta T_{R E}\left(X, X^{\prime}\right)$. Let $x \in X$. Then $x \in P$ for some $P \in \pi(\alpha)$. By Theorem 1 , there is $Q \in \pi(\beta)$ such that $P \subseteq Q$ and $Q \beta_{*} \leq P \alpha_{*}$. Hence $x \in Q$. We can see that $x \beta=Q \beta_{*} \leq$ $P \alpha_{*}=x \alpha$. Similarly, we can show that $x \alpha \leq x \beta$. Therefore, $x \alpha=x \beta$. The converse is clear.

Theorem 4. Let $\alpha, \beta \in P_{R E}\left(X, X^{\prime}\right)$. Then $\alpha \mathcal{R} \beta$ if and only if $\alpha=\beta$.

Proof. Assume that $\alpha \mathcal{R} \beta$. Then $\beta \in \alpha P_{R E}\left(X, X^{\prime}\right)$ and $\alpha \in \beta P_{R E}\left(X, X^{\prime}\right)$. It is easy to see that $\operatorname{dom} \alpha=\operatorname{dom} \beta$. Let $x \in \operatorname{dom} \alpha$. Then $x \in P$ for some $P \in \pi(\alpha)$ and $P \cap \operatorname{dom} \beta \neq \emptyset$. By Theorem 2, there is $Q \in \pi(\beta)$ such that $P \subseteq Q$ and $Q \beta_{*} \leq P \alpha_{*}$. Hence $x \in Q$. We can see that $x \beta=Q \beta_{*} \leq P \alpha_{*}=x \alpha$. Similarly, we can show that $x \alpha \leq x \beta$. Therefore, $x \alpha=x \beta$. The converse is clear.

Theorem 5. Let $\alpha, \beta \in T_{R E}\left(X, X^{\prime}\right)$. Then the following statements are equivalent.

1. $\alpha \in T_{R E}\left(X, X^{\prime}\right) \beta$.

2. For every $P \in \pi(\alpha)$, there exists $Q \in \pi(\beta)$ such that $Q \cap X^{\prime} \neq \emptyset$ and $P \alpha_{*}=Q \beta_{*}$ and for every $x \in P, d_{x} \leq x$ for some $d_{x} \in Q \cap X^{\prime}$.

Proof. Suppose that $\alpha=T_{R E}\left(X, X^{\prime}\right) \beta$. Then $\alpha=\gamma \beta$ for some $\gamma \in$ $T_{R E}\left(X, X^{\prime}\right)$. Let $P \in \pi(\alpha)$. Then $P \alpha_{*}=z$ for some $z \in X \alpha=X \gamma \beta \subseteq X \beta$. We obtain $z=x \gamma \beta$ for some $x \in X$. Let $Q=z \beta^{-1}$. We obtain $x \gamma \in z \beta^{-1} \cap X \gamma=$ $Q \cap X \gamma \neq \emptyset$ which implies that $Q \cap X^{\prime} \neq \emptyset$. Then $P \alpha_{*}=z=Q \beta_{*}$. Let $x \in P$. Then $x \gamma \beta=x \alpha=z$ which implies that $x \gamma \in Q \cap X^{\prime}$ and $x \gamma \leq x$.

Conversely, suppose that (2) holds. For each $x \in \operatorname{dom} \alpha$, there exists unique $P_{x} \in \pi(\alpha)$ such that $x \in P_{x}$. Then there exist $Q_{x} \in \pi(\beta)$ and $d_{x} \in Q_{x} \cap X^{\prime}$ such that $P_{x} \alpha_{*}=Q_{x} \beta_{*}$ and $d_{x} \leq x$. Define $\gamma:$ dom $\alpha \rightarrow X^{\prime}$ by $x \mapsto d_{x}$. We obtain $\gamma \in T_{R E}\left(X, X^{\prime}\right)$ and $x \gamma \beta=d_{x} \beta=Q_{x} \beta_{*}=P_{x} \alpha_{*}=x \alpha$. Therefore, $\alpha \in T_{R E}\left(X, X^{\prime}\right) \beta$. 
By the same proof as above, we have the following theorem immediately.

Theorem 6. Let $\alpha, \beta \in P_{R E}\left(X, X^{\prime}\right)$. Then the following statements are equivalent.

1. $\alpha \in P_{R E}\left(X, X^{\prime}\right) \beta$.

2. For every $P \in \pi(\alpha)$, there exists $Q \in \pi(\beta)$ such that $Q \cap X^{\prime} \neq \emptyset$ and $P \alpha_{*}=Q \beta_{*}$ and for every $x \in P, d_{x} \leq x$ for some $d_{x} \in Q \cap X^{\prime}$.

Theorem 7. Let $\alpha, \beta \in T_{R E}\left(X, X^{\prime}\right)$. Then the following statements are equivalent.

1. $\alpha \mathcal{L} \beta$.

2. $X \beta \subseteq X^{\prime} \alpha$ and for every $P \in \pi(\alpha)$ such that $P \cap X^{\prime} \neq \emptyset$, there exists $Q \in \pi(\beta)$ such that $Q \cap X^{\prime} \neq \emptyset$ and $P \alpha_{*}=Q \beta_{*}$ and for every $p \in P$ and $q \in Q$, there exist $a \in P \cap X^{\prime}$ and $b \in Q \cap X^{\prime}$ such that $b \leq p$ and $a \leq q$.

Proof. Assume that (1) holds. Then $\alpha=\gamma \beta$ and $\beta=\delta \alpha$ for some $\gamma, \delta \in$ $T_{R E}\left(X, X^{\prime}\right)$. We obtain $X \beta=X \delta \alpha \subseteq X^{\prime} \alpha$. Let $P \in \pi(\alpha)$. Since $\alpha=\gamma \beta \in$ $T_{R E}\left(X, X^{\prime}\right) \beta$, by Theorem 5, there is $Q \in \pi(\beta)$ such that $Q \cap X^{\prime} \neq \emptyset$ and $P \alpha_{*}=Q \beta_{*}$ and for each $p \in P, b \leq p$ for some $b \in Q \cap X^{\prime}$. Similarly, we obtain there is $P^{\prime} \in \pi(\alpha)$ such that $P^{\prime} \cap X^{\prime} \neq \emptyset$ and $P^{\prime} \alpha_{*}=Q \beta_{*}$ and for each $q \in Q, a \leq q$ for some $a \in P^{\prime} \cap X^{\prime}$. Since $P \alpha_{*}=Q \beta_{*}=P^{\prime} \alpha_{*}$, we get $P=P^{\prime}$ which implies that (2) holds.

Conversely, suppose that (2) holds. Then we obtain $\alpha \in T_{R E}\left(X, X^{\prime}\right) \beta$ by Theorem 5. To show that $\beta \in T_{R E}\left(X, X^{\prime}\right) \alpha$, let $Q \in \pi(\beta)$. Then $Q \beta_{*} \in$ $X \beta \subseteq X^{\prime} \alpha$ from which it follows that $Q \beta_{*}=P \alpha_{*}$ for some $P \in \pi(\alpha)$. By the assumption, there exists $Q^{\prime} \in \pi(\beta)$ such that $Q^{\prime} \cap X^{\prime} \neq \emptyset$ and $P \alpha_{*}=Q^{\prime} \beta_{*}$ and for every $q \in Q^{\prime}, a \leq q$ for some $a \in P \cap X^{\prime}$. We can see that $Q \beta_{*}=P \alpha_{*}=Q^{\prime} \beta_{*}$ which implies that $Q=Q^{\prime}$. Therefore, $\beta \in T_{R E}\left(X, X^{\prime}\right) \alpha$ by Theorem 5 .

By using the same proof as Theorem 7 , we obtain the result for $P_{R E}\left(X, X^{\prime}\right)$ as follows.

Theorem 8. Let $\alpha, \beta \in P_{R E}\left(X, X^{\prime}\right)$. Then the following statements are equivalent.

1. $\alpha \mathcal{L} \beta$.

2. $X \beta \subseteq X^{\prime} \alpha$ and for every $P \in \pi(\alpha)$ such that $P \cap X^{\prime} \neq \emptyset$, there exists $Q \in \pi(\beta)$ such that $Q \cap X^{\prime} \neq \emptyset$ and $P \alpha_{*}=Q \beta_{*}$ and for every $p \in P$ and $q \in Q$, there exist $a \in P \cap X^{\prime}$ and $b \in Q \cap X^{\prime}$ such that $b \leq p$ and $a \leq q$. 
Since $\mathcal{D}=\mathcal{L} \circ \mathcal{R}$ and $\mathcal{H}=\mathcal{L} \cap \mathcal{R}$, we obtain the following two theorems.

Corollary 9. Let $\alpha, \beta \in T_{R E}\left(X, X^{\prime}\right)$. Then the following statements hold.

1. $\alpha \mathcal{D} \beta$ if and only if $\alpha \mathcal{L} \beta$.

2. $\alpha \mathcal{H} \beta$ if and only if $\alpha=\beta$.

Corollary 10. Let $\alpha, \beta \in P_{R E}\left(X, X^{\prime}\right)$. Then the following statements hold.

1. $\alpha \mathcal{D} \beta$ if and only if $\alpha \mathcal{L} \beta$.

2. $\alpha \mathcal{H} \beta$ if and only if $\alpha=\beta$.

In the last part, we will characterize $\mathcal{J}$-relation on $T_{R E}\left(X, X^{\prime}\right)$ and $P_{R E}\left(X, X^{\prime}\right)$. Firstly, we state the following lemma which appears in [3].

Lemma 11. [3] If $\alpha, \beta, \delta, \gamma \in T(X)$ are such that $\alpha=\delta \beta \gamma$, then

$$
\mathcal{A}=\left\{\cup \mathcal{A}_{Q}: Q \in \pi(\beta) \text { and } Q \cap X \delta \neq \emptyset\right\}
$$

is a refinement of $\pi(\alpha)$ where $\mathcal{A}_{Q}=\left\{P \in \pi(\delta): P \delta_{*} \in Q\right\}$.

Now, we extend the above lemma to the partial transformation semigroup $P(X)$.

Lemma 12. If $\alpha, \beta, \delta, \gamma \in P(X)$ are such that $\alpha=\delta \beta \gamma$, then

$$
\mathcal{A}=\left\{\cup \mathcal{A}_{Q}: Q \in \pi(\beta), \cup \mathcal{A}_{Q} \neq \emptyset \text { and } Q \cap X \delta \neq \emptyset\right\}
$$

is a refinement of $\pi(\alpha)$ where $\mathcal{A}_{Q}=\left\{P \cap \operatorname{dom} \alpha: P \in \pi(\delta)\right.$ and $\left.P \delta_{*} \in Q\right\}$.

Proof. Let $\alpha, \beta, \delta, \gamma \in P(X)$ be such that $\alpha=\delta \beta \gamma$. We first show that $\cup \mathcal{A}=\cup \pi(\alpha)=\operatorname{dom} \alpha$. Let $x \in \operatorname{dom} \alpha \subseteq \operatorname{dom} \gamma$. Then $x \in P$ for some $P \in \pi(\delta)$. Since $x \delta \beta \in X \beta$, we obtain $x \delta \beta=Q \beta_{*}$ for some $Q \in \pi(\beta)$. Then $P \delta_{*}=x \delta \in Q$ which implies that $Q \cap X \delta \neq \emptyset$. Hence $P \cap \operatorname{dom} \alpha \in \mathcal{A}_{Q}$ and $x \in P \cap \operatorname{dom} \alpha \subseteq \cup \mathcal{A}_{Q} \subseteq \cup \mathcal{A}$. Thus dom $\alpha \subseteq \cup \mathcal{A}$. By the definition of $\mathcal{A}$, it is easy to see that $\cup \mathcal{A} \subseteq \operatorname{dom} \alpha$. Let $Q \in \pi(\beta)$ be such that $\cup \mathcal{A}_{Q} \neq \emptyset$ and $Q \cap X \delta \neq \emptyset$. We will prove that there is $\tilde{P} \in \pi(\alpha)$ such that $\cup \mathcal{A}_{Q} \subseteq \tilde{P}$. Let $x \in Q \cap X \delta$. Then there exists $y \in X$ such that $y \delta=x$. We obtain $y \in P$ for some $P \in \pi(\delta)$ and $P \delta_{*}=y \delta$. Since $\cup \mathcal{A}_{Q}$ is nonempty, there is $z \in \cup \mathcal{A}_{Q}$. Then $z \in P^{\prime} \cap \operatorname{dom} \alpha$ for some $P^{\prime} \in \pi(\delta)$ and $P^{\prime} \delta_{*} \in Q$. We can see that

$$
z \delta \beta=P^{\prime} \delta_{*} \beta=Q \beta_{*}=x \beta=y \delta \beta .
$$


Then

$$
z \alpha=z \delta \beta \gamma=y \delta \beta \gamma=y \alpha .
$$

Hence $y \in \operatorname{dom} \alpha$ which implies that $y \in \tilde{P}$ for some $\tilde{P} \in \pi(\alpha)$. Let $a \in \cup \mathcal{A}_{Q}$. Then $a \in \bar{P} \cap$ dom $\alpha$ for some $\bar{P} \in \pi(\delta)$ and $\bar{P} \delta_{*} \in Q$. We obtain

$$
a \alpha=a \delta \beta \gamma=\bar{P} \delta_{*} \beta \gamma=Q \beta_{*} \gamma=y \delta \beta \gamma=y \alpha=\tilde{P} \alpha_{*}
$$

Thus $a \in \tilde{P}$ from which it follows that $\cup \mathcal{A}_{Q} \subseteq \tilde{P}$. Therefore, $\mathcal{A}$ refines $\pi(\alpha)$.

Theorem 13. Let $\alpha, \beta \in T_{R E}\left(X, X^{\prime}\right)$. Then the following statements are equivalent.

1. $\alpha \in T_{R E}\left(X, X^{\prime}\right) \beta T_{R E}\left(X, X^{\prime}\right)$.

2. There exist a refinement $\mathcal{B}$ of $\pi(\alpha)$ and an injection $\varphi: \mathcal{B} \rightarrow \tilde{\pi}(\beta)$ such that for every $P \in \mathcal{B}, \tilde{P} \alpha_{*} \leq P \varphi \tilde{\beta}$ where $P \subseteq \tilde{P}$ and $\tilde{P} \in \pi(\alpha)$ and for every $x \in P, y \leq x$ for some $y \in P \varphi$.

Proof. Suppose that (1) holds. Then $\alpha=\delta \beta \gamma$ for some $\delta, \gamma \in T_{R E}\left(X, X^{\prime}\right)$. We obtain there is a refinement $\mathcal{A}$ of $\pi(\alpha)$ as defined in Lemma 11. Define $\varphi: \mathcal{A} \rightarrow \tilde{\pi}(\beta)$ by $\left(\cup A_{Q}\right) \varphi=Q \cap X^{\prime}$. Since $\mathcal{A}$ is a partition of dom $\alpha$, we get $\varphi$ is well-defined. Suppose that $\left(\cup \mathcal{A}_{Q}\right) \varphi=\left(\cup \mathcal{A}_{Q^{\prime}}\right) \varphi$. Then $Q \cap X^{\prime}=Q^{\prime} \cap X^{\prime}$ which implies that $Q=Q^{\prime}$ and $\cup \mathcal{A}_{Q}=\cup \mathcal{A}_{Q^{\prime}}$. Thus $\varphi$ is an injection.

Let $\cup \mathcal{A}_{Q} \in \mathcal{A}$. Then $Q \in \pi(\beta)$ and $Q \cap X \delta \neq \emptyset$. Let $x \in \cup \mathcal{A}_{Q}$. Then $x \in P$ for some $P \in \pi(\delta)$ such that $P \delta_{*} \in Q$. Hence $x \delta \in Q \cap X^{\prime}=\left(\cup \mathcal{A}_{Q}\right) \varphi$. Since $\mathcal{A}$ is a refinement of $\pi(\alpha)$, we get $x \in \cup \mathcal{A}_{Q} \subseteq \tilde{P}$ for some $\tilde{P} \in \pi(\alpha)$. We can see that

$$
\tilde{P} \alpha_{*}=x \alpha=x \delta \beta \gamma=Q \beta_{*} \gamma \leq Q \beta_{*}=\left(\cup \mathcal{A}_{Q}\right) \varphi \tilde{\beta} .
$$

Since $\delta$ is regressive, $x \delta \leq x$. In addition, $x \delta \in\left(\cup \mathcal{A}_{Q}\right) \varphi$. Therefore, (2) holds.

Conversely, suppose that (2) holds. For each $x \in \operatorname{dom} \alpha$, there is $P_{x} \in \mathcal{B}$ such that $x \in P_{x}$. Choose $y_{x} \in P_{x} \varphi$ such that $y_{x} \leq x$ and define $\delta: \operatorname{dom} \alpha \rightarrow X^{\prime}$ by $x \delta=y_{x}$. Then $\delta \in T_{R E}\left(X, X^{\prime}\right)$. We can see that $\varphi \tilde{\beta}: \mathcal{B} \rightarrow X \beta$ is injective since $\tilde{\beta}$ and $\varphi$ are injective. For each $x \in \mathcal{B} \varphi \tilde{\beta}$, there exists unique $P_{x}^{\prime} \in \mathcal{B}$ such that $x=P_{x}^{\prime} \varphi \tilde{\beta}$. By (2), there is $\tilde{P}_{x}^{\prime} \in \pi(\alpha)$ such that $\tilde{P}^{\prime}{ }_{x} \alpha_{*} \leq P_{x}^{\prime} \varphi \tilde{\beta}$ and $P_{x}^{\prime} \subseteq \tilde{P}_{x}^{\prime}$. Define $\gamma: X \rightarrow X^{\prime}$ by

$$
x \gamma= \begin{cases}\tilde{P}^{\prime}{ }_{x} \alpha_{*} & \text { if } \quad x \in \mathcal{B} \varphi \tilde{\beta} \\ x^{\prime} & \text { otherwise. }\end{cases}
$$

By the assumption, we obtain if $x \in \mathcal{B} \varphi \tilde{\beta}$, then $x \gamma=\tilde{P}^{\prime}{ }_{x} \alpha_{*} \leq\left(P_{x}^{\prime} \varphi\right) \tilde{\beta}=x$ and if $x \notin \mathcal{B} \varphi \tilde{\beta}$, then $x \gamma=x^{\prime} \leq x$. Hence $\gamma \in T_{R E}\left(X, X^{\prime}\right)$. Now, we show that 
$\alpha=\delta \beta \gamma$. Let $x \in \operatorname{dom} \alpha$. Then $x \in P_{x}$ for some $P_{x} \in \mathcal{B}$. By the definition of $\delta$, we have $x \delta=y_{x}$ where $y_{x} \in P_{x} \varphi$ which implies that $y_{x} \beta=P_{x} \varphi \tilde{\beta} \in \mathcal{B} \varphi \tilde{\beta}$. Hence $x \delta \beta \gamma=y_{x} \beta \gamma=\tilde{P}_{y_{x} \beta}^{\prime} \alpha_{*}$ where $\tilde{P}_{y_{x} \beta}^{\prime} \in \pi(\alpha)$ and $P_{x} \subseteq \tilde{P}_{y_{x} \beta}^{\prime}$ from which it follows that $x \alpha=\tilde{P}^{\prime}{ }_{y_{x}} \alpha_{*}$. Therefore, $x \delta \beta \gamma=x \alpha$.

By the same proof as the above theorem, we obtain the following result for $P_{R E}\left(X, X^{\prime}\right)$.

Theorem 14. Let $\alpha, \beta \in P_{R E}\left(X, X^{\prime}\right)$. Then the following statements are equivalent.

1. $\alpha \in P_{R E}\left(X, X^{\prime}\right) \beta P_{R E}\left(X, X^{\prime}\right)$.

2. There exist a refinement $\mathcal{B}$ of $\pi(\alpha)$ and an injection $\varphi: \mathcal{B} \rightarrow \tilde{\pi}(\beta)$ such that for every $P \in \mathcal{B}, \tilde{P} \alpha_{*} \leq P \varphi \beta_{*}$ where $P \subseteq \tilde{P}$ and $\tilde{P} \in \pi(\alpha)$ and for every $x \in P, y \leq x$ for some $y \in P \varphi$.

We obtain the following two corollaries immediately.

Corollary 15. Let $\alpha, \beta \in T_{R E}\left(X, X^{\prime}\right)$. Then $\alpha \mathcal{J} \beta$ if and only if the following statements holds.

1. There exist a refinement $\mathcal{B}$ of $\pi(\alpha)$ and an injection $\varphi: \mathcal{B} \rightarrow \tilde{\pi}(\beta)$ such that for every $P \in \mathcal{B}, \tilde{P} \alpha_{*} \leq P \varphi \beta_{*}$ where $P \subseteq \tilde{P}$ and $\tilde{P} \in \pi(\alpha)$ and for every $x \in P, y \leq x$ for some $y \in P \varphi$.

2. There exist a refinement $\mathcal{B}^{\prime}$ of $\pi(\beta)$ and an injection $\varphi^{\prime}: \mathcal{B}^{\prime} \rightarrow \tilde{\pi}(\alpha)$ such that for every $Q \in \mathcal{B}^{\prime}, \tilde{Q} \beta_{*} \leq Q \varphi^{\prime} \alpha_{*}$ where $Q \subseteq \tilde{Q}$ and $\tilde{Q} \in \pi(\beta)$ and for every $x^{\prime} \in Q, y^{\prime} \leq x^{\prime}$ for some $y^{\prime} \in Q \varphi^{\prime}$.

Corollary 16. Let $\alpha, \beta \in P_{R E}\left(X, X^{\prime}\right)$. Then $\alpha \mathcal{J} \beta$ if and only if the following statements holds.

1. There exist a refinement $\mathcal{B}$ of $\pi(\alpha)$ and an injection $\varphi: \mathcal{B} \rightarrow \tilde{\pi}(\beta)$ such that for every $P \in \mathcal{B}, \tilde{P} \alpha_{*} \leq P \varphi \beta_{*}$ where $P \subseteq \tilde{P}$ and $\tilde{P} \in \pi(\alpha)$ and for every $x \in P, y \leq x$ for some $y \in P \varphi$.

2. There exist a refinement $\mathcal{B}^{\prime}$ of $\pi(\beta)$ and an injection $\varphi^{\prime}: \mathcal{B}^{\prime} \rightarrow \tilde{\pi}(\alpha)$ such that for every $Q \in \mathcal{B}^{\prime}, \tilde{Q} \beta_{*} \leq Q \varphi^{\prime} \alpha_{*}$ where $Q \subseteq \tilde{Q}$ and $\tilde{Q} \in \pi(\beta)$ and for every $x^{\prime} \in Q, y^{\prime} \leq x^{\prime}$ for some $y^{\prime} \in Q \varphi^{\prime}$. 


\section{Acknowledgments}

The author was supported by CMU Junior Research Fellowship Program.

\section{References}

[1] P. Jitjankarn and P. Udomkavanich, Regularity of some regressive transformation semigroups, East-West Journal of Mathematics, Special Vol. (2004), 111-118.

[2] Y. Kemprasit, Quasi-regular regressive transformation semigroups, Southeast Asian Bulletin of Mathematics, 26 (2003), 955-959.

[3] C. Namnak and E. Laysirikul, Regularity and Green's relations for the full regressive transformation semigroups, International Journal of Algebra, 6, 17-20 (2012), 919-925.

[4] P. Udomkavanich and P. Jitjankarn, Isomorphism theorems of regressive partial transformation semigroups, Italian Journal of Pure and Applied Mathematics, 18 (2005), 207-212.

[5] P. Udomkavanich and P. Jitjankarn, Some isomorphism theorems on regressive transformation semigroups, Southeast Asian Bulletin of Mathematics, 29, 3 (2005), 581.

[6] P. Udomkavanich and P. Jitjankarn, A classification of regressive transformation semigroups on chains, Semigroup Forum, 85, 3 (2012), 559-570. doi:10.1007/s00233-012-94387

[7] A. Umar, Semigroups of order-decreasing transformations: The isomorphism theorem, Semigroup Forum, 53, 1 (1996), 220-224. doi:10.1007/BF02574137 